\title{
Substructures in the Keplerian disc around the O-type (proto-)star G17.64+0.16
}

\author{
L. T. Maud ${ }^{1,2}$, R. Cesaroni ${ }^{3}$, M. S. N. Kumar ${ }^{4}$, V. M. Rivilla ${ }^{3}$, A .Ginsburg ${ }^{5, \star \star}$, P. D. Klaassen ${ }^{6}$, D. Harsono ${ }^{2}$, \\ Á. Sánchez-Monge ${ }^{7}$, A. Ahmadi ${ }^{8}$, V. Allen ${ }^{9, \star \star \star}$, M. T. Beltrán ${ }^{3}$, H. Beuther ${ }^{8}$, R. Galván-Madrid ${ }^{10}$, C. Goddi ${ }^{2,11}$, \\ M. G. Hoare ${ }^{12}$, M. R. Hogerheijde ${ }^{2,13}$, K. G. Johnston ${ }^{12}$, R. Kuiper ${ }^{14}$, L. Moscadelli ${ }^{3}$, T. Peters ${ }^{15}$, L. Testi ${ }^{1,3}$,
} F. F. S. van der Tak ${ }^{16,17}$, and W. J. de Wit ${ }^{18}$

(Affiliations can be found after the references)

Received 5 April 2019 / Accepted 16 June 2019

\begin{abstract}
We present the highest angular resolution $(\sim 20 \times 15$ mas- $44 \times 33 \mathrm{au})$ Atacama Large Millimeter/sub-millimeter Array (ALMA) observations that are currently possible of the proto-O-star G17.64+0.16 in Band 6. The Cycle 5 observations with baselines out to $16 \mathrm{~km}$ probe scales $<50$ au and reveal the rotating disc around G17.64+0.16, a massive forming O-type star. The disc has a ring-like enhancement in the dust emission that is especially visible as arc structures to the north and south. The Keplerian kinematics are most prominently seen in the vibrationally excited water line, $\mathrm{H}_{2} \mathrm{O} 5_{5,0}-6_{4,3} v_{2}=1\left(E_{\mathrm{u}}=3461.9 \mathrm{~K}\right)$. The mass of the central source found by modelling the Keplerian rotation is consistent with $45 \pm 10 M_{\odot}$. The $\mathrm{H} 30 \alpha(231.9 \mathrm{GHz})$ radio-recombination line and the $\mathrm{SiO}(5-4)$ molecular line were detected at up to the $\sim 10 \sigma$ level. The estimated disc mass is 0.6-2.6 $M_{\odot}$ under the optically thin assumption. Analysis of the Toomre Q parameter in the optically thin regime indicates that the disc stability is highly dependent on temperature. The disc currently appears stable for temperatures $>150 \mathrm{~K}$; this does not preclude that the substructures formed earlier through disc fragmentation.
\end{abstract}

Key words. stars: formation - stars: protostars - stars: massive - stars: winds, outflows - stars: pre-main sequence - submillimeter: stars

\section{Introduction}

Spiral arms or rings and gaps in the discs of solar-mass protostars are now common, detectable at both IR and millimeter wavelengths (ALMA Partnership 2015; Brandt et al. 2014; Andrews et al. 2016, 2018; Walsh et al. 2017; Monnier et al. 2019; de Boer et al. 2016). Ring-gap structures have been explained by planets, zonal flows, snow lines, or dust trapping (Nazari et al. 2019; Isella \& Turner 2018; Dipierro et al. 2015; Ruge et al. 2016; Zhang et al. 2015), while spiral structures could be caused by interactions with planets or stellar binaries, or they might be due to a gravitational instability of the disc itself (Benisty et al. 2017; Quillen et al. 2005; Meru et al. 2017; Mayer et al. 2016). In the context of massive star formation, only a handful of sources have been found to show convincing evidence of disc rotation on sub-1000 au scales (e.g. Johnston et al. 2015; Ilee et al. 2016; Ginsburg et al. 2018; Moscadelli \& Goddi 2014; Moscadelli et al. 2019; Zapata et al. 2019). The detection of discs and rotation can still be somewhat difficult to identify even in the few targets that have been probed at sub-100 au resolution. Work by Beuther et al. (2019) indicates a highly fragmented star formation region in G351.77-0.54, with 12 identified structures, and tentative evidence of rotation in a few cores, while Goddi et al. (2018) observed the W51 region and also indi-

\footnotetext{
* Data cubes of the source are only available at the CDS via anony mous ftp to cdsarc.u-strasbg.fr $(130.79 .128 .5)$ or via http: //cdsarc.u-strasbg.fr/viz-bin/qcat?J/A+A/627/L6.

$\star \star$ Jansky fellow.

$\star \star \star$ NASA postdoctoral program fellow.
}

cated a highly clustered complex environment with little if any evidence for stable discs.

Theoretical works involving the formation of massive stars must invoke accretion discs. Simulations typically result in large-scale (500-1000 au) spiral-like features or streamers that funnel the accretion flows (Meyer et al. 2018; Harries et al. 2017; Krumholz et al. 2009; Peters et al. 2010; Kuiper et al. 2011; Klassen et al. 2016; Rosen et al. 2016; Kuiper \& Hosokawa 2018), which themselves have recently been observed in a few cases (Maud et al. 2017; Izquierdo et al. 2018; Liu et al. 2015; Cesaroni et al. 2014). Multiple or binary systems are clearly predicted by these simulations, and the substructures, which might be spirals, rings, or fragmented discs around massive protostars, should be possible to image using ALMA (e.g. Jankovic et al. 2019; Meyer et al. 2018).

Studies indicate that over $70 \%$ of main-sequence OB stars (Sana et al. 2012; Almeida et al. 2017), and 50\% of massive young stellar objects (Pomohaci et al. 2019) are known to display binarity or multiplicity. In the deeply embedded star formation stages, very high angular resolution $(<100$ au scales) sub-millimeter observations are required to probe the natal environments. There is one recent example of a binary protoO-star system where the secondary, separated by $\sim 1200 \mathrm{au}$, is still within the disc and thought to have formed by disc fragmentation (Ilee et al. 2018). Furthermore, Zhang et al. (2019) presented ALMA long-baseline observations that resolved a high-mass binary system (total mass $\sim 18 M_{\odot}$ ) with a physical separation of $\sim 180 \mathrm{au}$. However, rings, gaps, or spiral substructures at spatial scales lower than 500 au have not yet been detected in discs around massive protostars. 

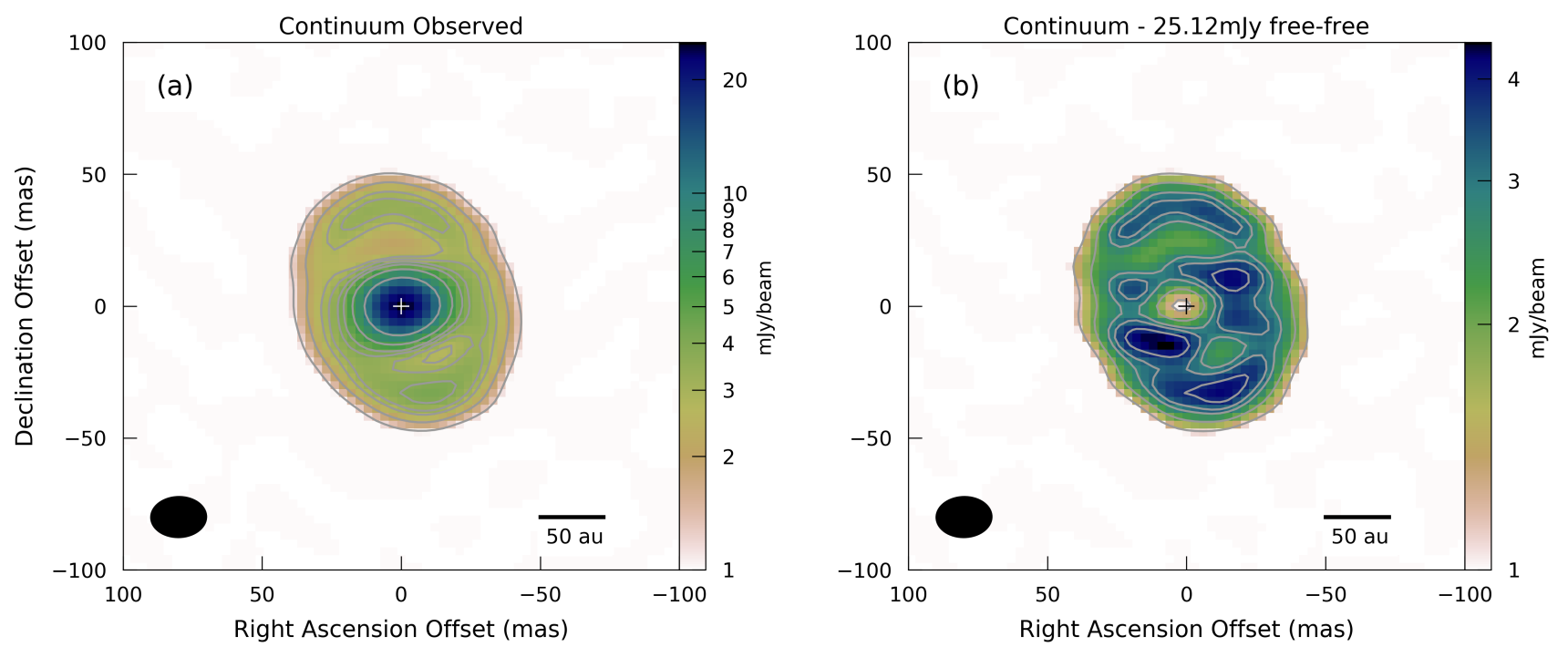

Fig. 1. Panel a: ALMA band $6(1.3 \mathrm{~mm})$ long-baseline continuum image of G17.64 made at a resolution of $20 \times 15$ mas, PA $-88.4^{\circ}$. The enhanced emission is clearest in the north and south between 65 and 97 au in the radial direction from G17.64. Panel b: same as (a), but with a point source of peak flux $25.12 \mathrm{mJy}_{\text {beam }}{ }^{-1}$ removed from the visibilities, representing the strongest free-free contamination in G17.64. Additional substructures now become clear. Note the change in the scale of the colour bar. All contours are drawn at 30, 50, 70, 80, 95, 110, 150, and 250 $\sigma$ of the respective images, where $1 \sigma=40.4 \mu \mathrm{Jy}_{\text {beam }}{ }^{-1}$. The beam and scale bar are indicated at the bottom.

The source G17.64+0.16 (hereafter G17.64, also AFGL 2136, G017.6380+00.1566, CRL 2136, and IRAS 18196-1331) is a well-known massive young stellar object (MYSO) that we originally targeted with ALMA along with five other luminous O-type (proto-)stars in search for evidence of discs (Cesaroni et al. 2017). Located at $2.2 \mathrm{kpc}$ and with a bolometric luminosity of $1 \times 10^{5} L_{\odot}{ }^{1}$ (Lumsden et al. 2013), G17.64 is one of the closest O-type (proto-)stars. It is a bright source at nearto mid-IR wavelengths (Kastner et al. 1992; Holbrook \& Temi 1998; de Wit et al. 2009; Murakawa et al. 2013) and is detected through to the centimeter regime (e.g. van der Tak et al. 2000; Menten \& van der Tak 2004; Lu et al. 2014). It drives an outflow (position angle, $\mathrm{PA} \sim 135^{\circ}$ ), illuminates a reflection nebulae, and excites $\mathrm{H}_{2} \mathrm{O}$ masers (Menten \& van der Tak 2004). Interferometric IR observations indicated that G17.64 is a candidate compact ( $<100 \mathrm{au}$ ) disc source (Boley et al. 2013). Maud et al. (2018) presented 0.2" resolution ALMA data probing scales down to $\sim 400$ au. They did not resolve the continuum emission, but marginally resolved the $\mathrm{SiO}$ emission that is thought to trace a rotating disc and disc wind. Modelling the position-velocity (PV) profile, they estimated a central source mass between 20 and $30 M_{\odot}$.

In this Letter we report on the observations of G17.64 using the ALMA long-baselines at band 6 . Achieving a resolution of $20 \times 15$ mas $(44 \times 33 \mathrm{au})$, which is ten times higher than our previous study, we now fully resolve the dust and molecular line emission and for the first time uncover clear enhanced substructures in the disc around this massive forming O-star.

\section{Observations}

The ALMA $12 \mathrm{~m}$ observations consist of one execution block (EB) conducted during Cycle 5 on 4 October 2017 (project ID: 2017.1.00098.S - PI: Maud) in configuration C43-10, with 43 antennas. The on-source time was $30.6 \mathrm{~min}$. The spectral setup covered the frequency range of our previous observations (see

\footnotetext{
1 Red MSX Survey: http://rms.leeds.ac.uk/cgi-bin/ public/RMS_DATABASE.cgi
}

also Cesaroni et al. 2017). To provide maximum sensitivity to the dust continuum, all four spectral windows (SPWs) were configured with the widest bandwidth of $1.875 \mathrm{GHz}$ but covered previously detected lines, such as $\mathrm{SiO}(5-4)$ and $\mathrm{H} 30 \alpha$. The resulting velocity resolution was of the order $1.3 \mathrm{~km} \mathrm{~s}^{-1}$, except in the SPW covering $\mathrm{SiO}$, which was $0.8 \mathrm{~km} \mathrm{~s}^{-1}$. We also covered the $\mathrm{H}_{2} \mathrm{O} 5_{5,0}-6_{4,3}$ line in the same SPW as $\mathrm{H} 30 \alpha$. The maximum angular resolution of $20 \times 15 \mathrm{mas}$ at a PA of $-88.4^{\circ}$ in the continuum was achieved using a robust parameter of 0.0 (Briggs 1995). Data calibration used the CASA pipeline, version 5.1.1 (McMullin et al. 2007), while subsequent imaging and self-calibration used version 5.4.0. Because G17.64 is relatively line weak (Cesaroni et al. 2017), all line-free regions were easily identifiable and continuum subtraction was undertaken in the $u, v$ domain. Self-calibration was possible down to a solution time of $6 \mathrm{~s}$ in phase and $54 \mathrm{~s}$ for amplitude, which improved the dynamic range from 405 to 640 . The final continuum noise level we achieved was $40.4 \mu \mathrm{Jy}_{\text {beam }}{ }^{-1}$. For the $\mathrm{H}_{2} \mathrm{O}$ images, a robust value of 1.5 was used to boost the surface brightness sensitivity. The resulting resolution was $28 \times 23$ mas at a PA of $65.8^{\circ}$ and achieved a sensitivity of $0.76 \mathrm{mJy} \mathrm{bm}^{-1} \mathrm{ch}^{-1}\left(1.3 \mathrm{~km} \mathrm{~s}^{-1}\right)$. We detected $\mathrm{SiO}(5-4)$ and the $\mathrm{H} 30 \alpha$ radio-recombination line, which are mentioned in the appendix. During self-calibration we shifted the phase centre of G17.64 to the position of peak emission, J2000 $18^{\mathrm{h}} 22^{\mathrm{m}} 26.3862^{\mathrm{s}}-13^{\circ} 30^{\prime} 11.9717^{\prime \prime}$, to centralise our images. All imaging and self-calibration steps were performed with and without shifting the phase centre to ensure that the detected features were real and not interferometric side-lobe artefacts. No notable differences were seen in any of our images.

\section{Results}

\subsection{Continuum emission}

Figure 1a shows our image of the continuum emission from G17.64 in a logarithmic colour scaling to highlight the fainter emission. The continuum dust disc is well resolved and has a clear, enhanced, ring-like structure that is most readily visible to the north and south between 65 and 97 au in the radial direction. 
Fitting an ellipse to the $50 \sigma$ contour level $(\sim 2.0 \mathrm{mJy}$ beam $\left.{ }^{-1}\right)$, we find that the disc measures $93 \times 71$ mas. Assuming a circular thin disc where the millimeter-sized dust grains have settled to the mid-plane (e.g. Testi et al. 2014), we estimate the disc inclination as $\sim 40 \pm 4^{\circ}$ (where $90^{\circ}$ is edge-on). The uncertainty propagates from fitting the disc size at the $30 \sigma$ and $70 \sigma$ contours and from calculation of the respective inclination angles. The peak flux is $25.12 \mathrm{mJy}_{\text {beam }}{ }^{-1}$, while the enhanced arc regions peak at between 3 and $4 \mathrm{mJy}^{-1}$ beam $^{-1}$. We recover an integrated flux of $79.14 \mathrm{mJy}$ (within a circle with a 60 mas radius), which is entirely consistent with the previous ALMA observations ( $81.3 \mathrm{mJy}$ before free-free subtraction, Maud et al. 2018) and confirms that we did not resolve out any emission and can attribute the total flux entirely to the disc. Using a minimum value of $250 \mathrm{~m}$ when considering the well-sampled short baselines, we note that our maximum recoverable scale is at least $0.64^{\prime \prime}$, much larger than the dust disc.

Maud et al. (2018) reported that the radio wavelength emission accounts for between $3.57 \mathrm{mJy}$ and $29.5 \mathrm{mJy}$ when it is extrapolated to the millimeter regime. When we consider that the peak flux is $25.12 \mathrm{mJy}$ beam $^{-1}$, this places an upper limit on the free-free contamination in the case that it fully represents the millimeter emission. In Fig. 1b we show the image of G17.64 after the subtraction of a point source, made in the visibility domain, using the upper limit of the free-free contamination. A point-source assumption is consistent with the size $(29$ mas at $43 \mathrm{GHz}$ ) found by Menten \& van der Tak (2004) and the reduction in angular size with increasing frequency $\left(\theta \propto v^{-0.6}\right.$, Wright \& Barlow 1975). The removal of a point source in the visibility domain can be understood as removing a 2D Gaussian with the synthesised beam parameters in the image plane. After the removal of the maximum and minimum free-free components, the resulting lower limit of the optically thin disc mass ranges from 0.6 to $2.6 M_{\odot}$ following Hildebrand (1983) and using dust temperatures of $50 \mathrm{~K}, 100 \mathrm{~K}$, and $150 \mathrm{~K}$ consistent with Maud et al. (2018) and Sect. 4.2 (see the appendix for details).

Jankovic et al. (2019) reported that the total removal of a central Gaussian feature can be a useful technique to highlight residual substructures. It is coincidental that the necessary removal of the free-free contamination from G17.64 has a similar effect. In Fig. 1b the brightest structure in the dust disc has a peak flux of $4.4 \mathrm{mJy}^{\text {beam }}{ }^{-1}$. Although speculative because the free-free contamination is uncertain, we tentatively reveal an enhanced inner ring or poorly resolved spiral-like structure.

\section{2. $\mathrm{H}_{2} \mathrm{O}$}

Figure 2 presents the zero-moment and first-moment maps of the $\mathrm{H}_{2} \mathrm{O} 5_{5,0}-6_{4,3} v_{2}=1$ line at $232.68670 \mathrm{GHz}\left(E_{\mathrm{u}}=3461.9 \mathrm{~K}\right)$ in panels $a$ and $b$ and PV diagrams over plotted with contours of the data and the best representative model in panels $\mathrm{c}$ and d. The extreme excitation energy of this line makes it particularly suited for tracing the very inner regions of a molecular disc (Hirota et al. 2014; Ginsburg et al. 2018). To our sensitivity and resolution limit, the $\mathrm{H}_{2} \mathrm{O}$ emission appears devoid of any structure and does not extend beyond the outer radius of the disc as traced by the dust continuum emission (grey contours Fig. 2b). The peak of emission is away from the centre of the dust disc in all velocity channels, while the $\mathrm{H}_{2} \mathrm{O}$ emission peaks between the enhanced continuum dust ring and the central location of G17.64 at the highest blue- and red-shifted velocities. The zeromoment contours in Fig. 2a show that the $\mathrm{H}_{2} \mathrm{O}$ emission might appear like a disc with a central hole or like a single wide ring ranging from approximately half of the beam ( $30 \mathrm{au})$ to $120 \mathrm{au}$ in radius (e.g. like the SO emission in Yen et al. 2014). The cut for the PV diagram was taken at a PA of $25.9^{\circ}$ using a width of 5 pixels to encompass one synthesised beam (grey dashed line in Figs. 2a,b). The PA was established by fitting a line through the centres of Gaussian fits to independent zero-moment maps of the blue- $\left(3.2-21.4 \mathrm{~km} \mathrm{~s}^{-1}\right)$ and red-shifted $\left(22.7-44.8 \mathrm{~km} \mathrm{~s}^{-1}\right)$ emission and the central position of G17.64 from the dust continuum. The PV diagram depicts the clear pattern of Keplerian rotation, particularly the red-shifted emission $>30 \mathrm{~km} \mathrm{~s}^{-1}$, where the highest velocities peak closer towards the central source. The $\mathrm{H}_{2} \mathrm{O}$ undoubtedly traces a rotating disc.

\section{Discussion}

\subsection{Stellar mass}

Based on its luminosity, G17.64 is firmly positioned as an O star (e.g. Vacca et al. 1996). However, previous estimates of the stellar mass, which are essential for understanding the disc stability, were not well constrained because the kinematics were poorly resolved (Maud et al. 2018). Building from our previous representative models presented in Maud et al. (2018), we matched the $\mathrm{H}_{2} \mathrm{O} \mathrm{PV}$ diagram with only very minor changes. We used our disc-only model for the $\mathrm{H}_{2} \mathrm{O}$ emission because there is no evidence for extended structure, we fixed the disc outer radius to $120 \mathrm{au}$ according to the dust and $\mathrm{H}_{2} \mathrm{O}$ maps and also revised the inclination angle to $40 \pm 5^{\circ}$. Now that the disc is fully resolved, we have a measure of the system inclination, which was a previously degenerate parameter (Maud et al. 2018). The inner radius was varied only between 25 and 30 au in line with the lack of strong $\mathrm{H}_{2} \mathrm{O}$ emission at the centre (Fig. 2a, see also Maud et al. 2018), and is consistent with the potentially ionised inner disc seen in $\mathrm{H} 30 \alpha$ emission (see the appendix). With these modifications, the modelled central mass must be increased to $45 \pm 10 M_{\odot}$ to best match the observed PV diagram (Figs. 2c,d), especially the red-shifted emission at higher velocities. The mass increase compared to Maud et al. (2018) is due to the revised inclination angle because the disc is seen more face-on. Following Maud et al. (2018), fitting was made by eye by matching equivalent contour levels (see also Ilee et al. 2018).

\subsection{Disc stability}

In discs undergoing Keplerian rotation, local regions may be unstable to axisymmetric perturbations if the Toomre parameter, $\mathrm{Q}$ (Toomre 1964), is

$Q=\frac{c_{\mathrm{s}} \kappa}{\pi G \Sigma}<1$,

where $c_{\mathrm{s}}$ is the sound speed, $\kappa$ is the epicyclic frequency (the orbital velocity $\Omega$ for a Keplerian disc), $G$ is the gravitational constant, and $\Sigma$ is the disc surface density. Figures 3a,b show the Toomre Q maps for G17.64 where the minimum and maximum free-free contamination is removed. A deprojection has been applied in the image plane for the minor disc axis after rotation (the major axis is north-south). We followed the methods outlined in Beuther et al. (2017) and Ahmadi et al. (2018). Both the mass surface density, calculated from the column density (Eq. (2), Schuller et al. 2009) multiplied by the mean molecular weight and mass of the hydrogen atom $\left(\mu m_{\mathrm{H}}\right.$, where $\mu=2.3$ ), and the sound speed use a radial temperature dependence, and we directly calculated the orbital velocity from Keplerian rotation. The temperature of the disc was calculated using $T(R) \propto\left(R / R_{0}\right)^{-0.4}$ (Whitney et al. 2003) propagating from the 

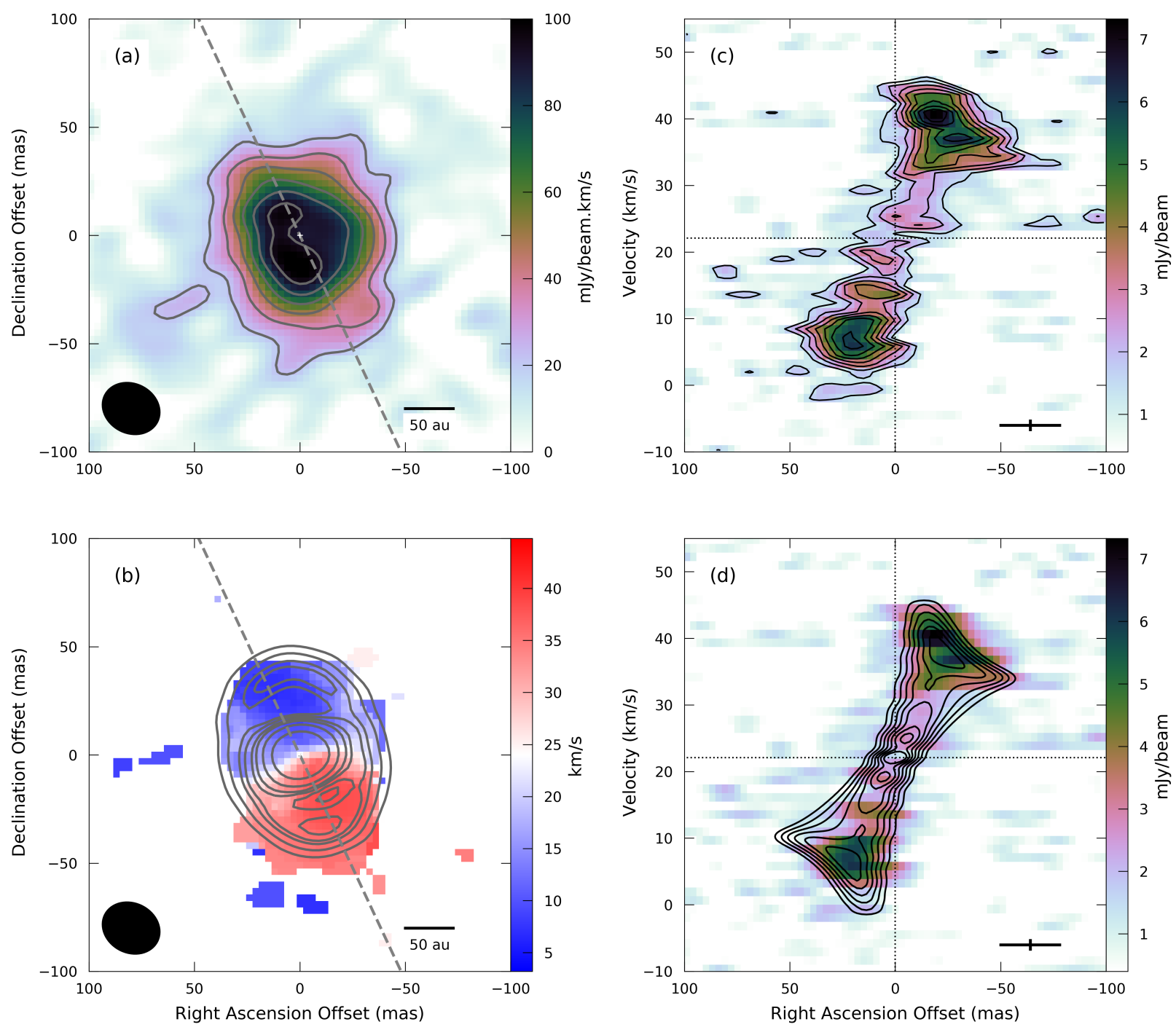

Fig. 2. Panel $a$ : integrated intensity (zero-moment) map of the $\mathrm{H}_{2} \mathrm{O} 5_{5,0}-6_{4,3} v_{2}=1(232.68670 \mathrm{GHz})$ molecular line emission. The contours trace emission at the $3,5,7,9$, and $11 \sigma$ levels, where $1 \sigma=7.13 \mathrm{mJy}_{\text {beam }}^{-1} \mathrm{~km} \mathrm{~s}^{-1}$. Panel $b$ : velocity-weighted integrated intensity (first-moment) map overlaid with the contours of the continuum emission from Fig.1a in grey. The outer extent of the $\mathrm{H}_{2} \mathrm{O}$ emission matches that of the dust continuum. Panel c: PV diagram for the $\mathrm{H}_{2} \mathrm{O}$ emission extracted from a 5 pixel wide slice along the dashed line at $\mathrm{PA}=25.9^{\circ}$ as indicated in (a) and (b). The contours of the data are plotted at the $20-80 \%$ levels in steps of $10 \%$, where $10 \%$ corresponds to $\sim 1 \sigma\left(0.65 \mathrm{mJy}^{-1}\right.$ beam $\left.{ }^{-1}\right)$. Panel $d$ : same as (c), but the contours indicate the best representative model using inner and outer disc radii of 30 and 120 au, respectively, a stellar mass of $45 M_{\odot}$, and a disc inclination angle of $40^{\circ}$. The spatial $(28 \mathrm{mas})$ and velocity resolution $\left(1.3 \mathrm{~km} \mathrm{~s}^{-1}\right)$ are indicated by the black cross at the bottom right.

outer radius and temperature of a $45 M_{\odot}$ main-sequence $\mathrm{O}$ star from Hosokawa \& Omukai (2009), where for G17.64 we estimated $R_{0} \sim 9.3 R_{\odot}, L \sim 1.9 \times 10^{5} L_{\odot}$ and $T \sim 60000 \mathrm{~K}$. At radii $<150$ au the disk temperature is $>150 \mathrm{~K}$.

The ring-like enhancement between radii 65 and 97 au has the lowest Toomre $\mathrm{Q}$ values, between 2 and 4 . There is little difference between the data with the minimum and maximum free-free contamination removed, respectively, because the lowest $\mathrm{Q}$ regions are away from the central peak where the freefree contamination occurs. No instabilities are identified in the substructures (cf. Fig. 1b). When we used a steeper temperature profile (e.g. - 0.5, Brinch \& Hogerheijde 2010), the temperature in the disc was below $150 \mathrm{~K}$, and the overall cooler disc could become unstable, $Q<1$. Additionally, in an optically thick case as suggested by the continuum average brightness temperatures (see the appendix), the surface density will increase when we correct for opacity and Q would decrease. If we were to consider a highly flared disc, the Toomre criterion would be met where $Q<1.7$ (Durisen et al. 2007), thus a proxy of the dust and gas disc scale heights and temperature estimation from radiative transfer are required to better constrain the stability. The presented Toomre Q analysis suggests that unless the dust is cold and significantly shielded, the disc can remain stable against fragmentation. This does not preclude that the existing substructures may have formed from fragmentation. During formation, a more massive, cooler, and potentially largely flared disc combined with a reduced stellar mass would have yielded conditions where $Q<1.7$.

\subsection{Disc enhancements}

The star G17.64 is the first massive O-type source in which substructure has been observed in the disc. Interestingly, recent IR interferometry and models by Frost et al. (2019) suggest that 

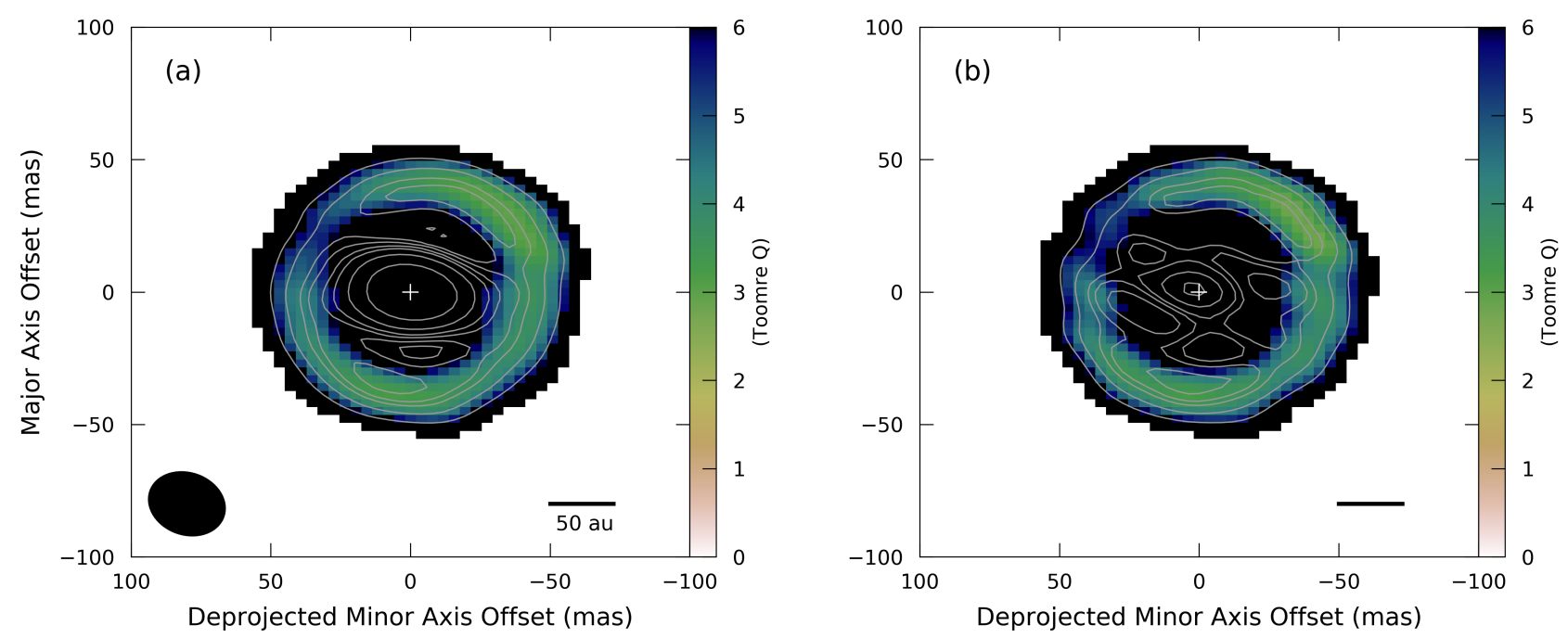

Fig. 3. Panel a: Toomre Q map of G17.64 using the dust emission with the minimum free-free fraction subtracted for the mass surface density. Panel b: same as (a), but the maximum free-free contamination was removed before the mass surface density was calculated. The synthesised beam is indicated at the bottom left of the left figure and a scale to the right, while the grey contours are those shown in Figs. 1a and b. Values below the $10 \sigma$ dust continuum emission level are masked out. The maps are centred $(0,0)$ on G17.64 but are rotated by the PA $\left(25.9^{\circ}\right)$, aligning the disc major axis in the north-south direction. The disc is deprojected along the minor axis using the inclination of $40^{\circ}$ in the image plane.

the MYSO G305.20+0.21 could be a massive transition disc, with an inner edge at $60 \mathrm{au}$, similar to the ring-enhancement in G17.64, although there are no high-resolution millimeter studies. Similar processes, such as radial drifts, could be at work in G17.64 as they are in low-mass sources (Pinilla et al. 2018). In this case, dust grains become trapped in pressure maxima and can grow (see Sect. 1), but multi-wavelength observations are required to establish dust grain sizes and confirm the phenomena. When we consider that most massive systems exist as binaries, it is plausible that a binary companion is the cause of the ring-like enhancements, much like the $1.8 M_{\odot}$ source HD 142527 (Price et al. 2018). We could interpret the brightest dust peak south-east of G17.64+0.16 as a binary companion, although there is no clearly separated source like in Ilee et al. (2018). The substructures could be spirals caused by instabilities that occurred before this stable disc phase, which would appear as arc- or ring-like enhancements if not fully resolved. Higher frequency observations may be the only way to probe $10 \mathrm{au}$ scales, provided that substructures are still visible with increasing optical depth.

\section{Conclusions}

We presented ALMA long-baseline observations that reveal the disc in the proto-O-star G17.64+0.16. Through the PV analysis of the $\mathrm{H}_{2} \mathrm{O}$ emission using our disc model, we confirm Keplerian rotation about a central mass of $45 \pm 10 M_{\odot}$. We find that the continuum emission has a significant enhancement of dust emission in a ring-like or possibly underlying spiral structure. The disc is found to be locally stable through a Toomre $\mathrm{Q}$ analysis in the optically thin case when disc temperatures are $>150 \mathrm{~K}$.

Acknowledgements. The authors thank the referee for their detailed comments that helped to improve this manuscript. MSNK acknowledges the support from Fundação para a Ciência e Tecnologia through Investigador contracts and exploratory project IF/00956/2015/CP1273/CT0002. HB and AA acknowledge support from the European Research Council under the European Community's Horizon 2020 framework program (2014-2020) via the ERC Consolidator Grant “From Cloud to Star Formation (CSF)" (project number 648505). RGM acknowledges support from UNAM-PAPIIT Programme IN104319. RK acknowledges financial support via the Emmy Noether Research Group funded by the German Research Foundation (DFG) under grant no. KU 2849/3-1 and KU 2849/3-2. VMR thanks the funding from the European Union's Horizon 2020 research and innovation programme under the Marie Skłodowska-Curie grant agreement No 664931. This paper makes use of the least-squares fitting by Hammel \& Sullivan-Molina (2019). This paper makes use of the following ALMA data: ADS/JAO.ALMA\#2017.1.00098.S. ALMA is a partnership of ESO (representing its member states), NSF (USA) and NINS (Japan), together with NRC (Canada), NSC and ASIAA (Taiwan), and KASI (Republic of Korea), in cooperation with the Republic of Chile. The Joint ALMA Observatory is operated by ESO, AUI/NRAO and NAOJ.

\section{References}

Ahmadi, A., Beuther, H., Mottram, J. C., et al. 2018, A\&A, 618, A46 ALMA Partnership, Brogan, C. L., Pérez, L. M., et al. 2015, ApJ, 808, L3 Almeida, L. A., Sana, H., Taylor, W., et al. 2017, A\&A, 598, A84 Andrews, S. M., Wilner, D. J., Zhu, Z., et al. 2016, ApJ, 820, L40 Andrews, S. M., Huang, J., Pérez, L. M., et al. 2018, ApJ, 869, L41 Benisty, M., Stolker, T., Pohl, A., et al. 2017, A\&A, 597, A42 Beuther, H., Walsh, A. J., Johnston, K. G., et al. 2017, A\&A, 603, A10 Beuther, H., Ahmadi, A., Mottram, J. C., et al. 2019, A\&A, 621, A122 Boley, P. A., Linz, H., van Boekel, R., et al. 2013, A\&A, 558, A24 Brandt, T. D., Kuzuhara, M., McElwain, M. W., et al. 2014, ApJ, 786, 1 Briggs, D. S. 1995, in American Astronomical Society Meeting Abstracts, Bull. Am. Astron. Soc., 27, 1444

Brinch, C., \& Hogerheijde, M. R. 2010, A\&A, 523, A25

Cesaroni, R., Galli, D., Neri, R., \& Walmsley, C. M. 2014, A\&A, 566, A73 Cesaroni, R., Sánchez-Monge, Á., Beltrán, M. T., et al. 2017, A\&A, 602, A59 de Boer, J., Salter, G., Benisty, M., et al. 2016, A\&A, 595, A114

de Wit, W. J., Hoare, M. G., Fujiyoshi, T., et al. 2009, A\&A, 494, 157

Dipierro, G., Pinilla, P., Lodato, G., \& Testi, L. 2015, MNRAS, 451, 974

Durisen, R. H., Boss, A. P., Mayer, L., et al. 2007, in Protostars and Planets V, eds. B. Reipurth, D. Jewitt, \& K. Keil, 607

Frost, A. J., Oudmaijer, R. D., de Wit, W. J., \& Lumsden, S. L. 2019, A\&A, 625, A44

Ginsburg, A., Bally, J., Goddi, C., Plambeck, R., \& Wright, M. 2018, ApJ, 860, 119

Goddi, C., Ginsburg, A., Maud, L., Zhang, Q., \& Zapata, L. 2018, ArXiv e-prints [arXiv:1805.05364]

Hammel, B., \& Sullivan-Molina, N. 2019, https://doi.org/10.5281/zenodo. 2578663

Harries, T. J., Douglas, T. A., \& Ali, A. 2017, MNRAS, 471, 4111

Hildebrand, R. H. 1983, Q. J. R. Astron. Soc., 24, 267

Hirota, T., Kim, M. K., Kurono, Y., \& Honma, M. 2014, ApJ, 782, L28

Holbrook, J. C., \& Temi, P. 1998, ApJ, 496, 280

Hosokawa, T., \& Omukai, K. 2009, ApJ, 691, 823 
Ilee, J. D., Cyganowski, C. J., Nazari, P., et al. 2016, MNRAS, 462, 4386 Ilee, J. D., Cyganowski, C. J., Brogan, C. L., et al. 2018, ApJ, 869, L24 Isella, A., \& Turner, N. J. 2018, ApJ, 860, 27

Izquierdo, A. F., Galván-Madrid, R., Maud, L. T., et al. 2018, MNRAS, 478 , 2505

Johnston, K. G., Robitaille, T. P., Beuther, H., et al. 2015, ApJ, 813, L19

Jankovic, M. R., Haworth, T. J., Ilee, J. D., et al. 2019, MNRAS, 482, 4673

Kastner, J. H., Weintraub, D. A., \& Aspin, C. 1992, ApJ, 389, 357

Klassen, M., Pudritz, R. E., Kuiper, R., Peters, T., \& Banerjee, R. 2016, ApJ, 823, 28

Krumholz, M. R., Klein, R. I., McKee, C. F., Offner, S. S. R., \& Cunningham, A. J. 2009, Science, 323, 754

Kuiper, R., \& Hosokawa, T. 2018, A\&A, 616, A101

Kuiper, R., Klahr, H., Beuther, H., \& Henning, T. 2011, ApJ, 732, 20

Liu, H. B., Galván-Madrid, R., Jiménez-Serra, I., et al. 2015, ApJ, 804, 37

Lu, X., Zhang, Q., Liu, H. B., Wang, J., \& Gu, Q. 2014, ApJ, 790, 84

Lumsden, S. L., Hoare, M. G., Urquhart, J. S., et al. 2013, ApJS, 208, 11

Maud, L. T., Hoare, M. G., Galván-Madrid, R., et al. 2017, MNRAS, 467, L120

Maud, L. T., Cesaroni, R., Kumar, M. S. N., et al. 2018, A\&A, 620, A31

Mayer, L., Peters, T., Pineda, J. E., Wadsley, J., \& Rogers, P. 2016, ApJ, 823, L36

McMullin, J. P., Waters, B., Schiebel, D., Young, W., \& Golap, K. 2007, in Astronomical Data Analysis Software and Systems XVI, eds. R. A. Shaw, F. Hill, \& D. J. Bell, ASP Conf. Ser., 376, 127

Menten, K. M., \& van der Tak, F. F. S. 2004, A\&A, 414, 289

Meru, F., Juhász, A., Ilee, J. D., et al. 2017, ApJ, 839, L24

Meyer, D. M. A., Kuiper, R., Kley, W., Johnston, K. G., \& Vorobyov, E. 2018, MNRAS, 473, 3615

Monnier, J. D., Harries, T. J., Bae, J., et al. 2019, ApJ, 872, 122

Moscadelli, L., \& Goddi, C. 2014, A\&A, 566, A150

Moscadelli, L., Sanna, A., Cesaroni, R., et al. 2019, A\&A, 622, A206

Murakawa, K., Lumsden, S. L., Oudmaijer, R. D., et al. 2013, MNRAS, 436, 511

Nazari, P., Booth, R. A., Clarke, C. J., et al. 2019, MNRAS, 485, 5914

Ossenkopf, V., \& Henning, T. 1994, A\&A, 291, 943

Peters, T., Mac Low, M.-M., Banerjee, R., Klessen, R. S., \& Dullemond, C. P. 2010, ApJ, 719, 831

Pinilla, P., Tazzari, M., Pascucci, I., et al. 2018, ApJ, 859, 32

Pomohaci, R., Oudmaijer, R. D., \& Goodwin, S. P. 2019, MNRAS, 484, 226

Price, D. J., Cuello, N., Pinte, C., et al. 2018, MNRAS, 477, 1270

Quillen, A. C., Varnière, P., Minchev, I., \& Frank, A. 2005, AJ, 129, 2481

Rosen, A. L., Krumholz, M. R., McKee, C. F., \& Klein, R. I. 2016, MNRAS, 463,2553

Ruge, J. P., Flock, M., Wolf, S., et al. 2016, A\&A, 590, A17

Sana, H., de Mink, S. E., de Koter, A., et al. 2012, Science, 337, 444

Schuller, F., Menten, K. M., Contreras, Y., et al. 2009, A\&A, 504, 415

Testi, L., Birnstiel, T., Ricci, L., et al. 2014, in Protostars and Planets VI, eds. H.

Beuther, R. S. Klessen, C. P. Dullemond, \& T. Henning, 339

Toomre, A. 1964, ApJ, 139, 1217

Vacca, W. D., Garmany, C. D., \& Shull, J. M. 1996, ApJ, 460, 914 van der Tak, F. F. S., van Dishoeck, E. F., Evans, II., N. J., \& Blake, G. A. 2000, ApJ, 537, 283

Walsh, C., Daley, C., Facchini, S., \& Juhász, A. 2017, A\&A, 607, A114

Whitney, B. A., Wood, K., Bjorkman, J. E., \& Wolff, M. J. 2003, ApJ, 591, 1049 Wright, A. E., \& Barlow, M. J. 1975, MNRAS, 170, 41

Yen, H.-W., Takakuwa, S., Ohashi, N., et al. 2014, ApJ, 793, 1

Zapata, L. A., Garay, G., Palau, A., et al. 2019, ApJ, 872, 176

Zhang, K., Blake, G. A., \& Bergin, E. A. 2015, ApJ, 806, L7

Zhang, Y., Tan, J. C., Tanaka, K. E. I., et al. 2019, Nat. Astron., 224

1 European Southern Observatory, Karl-Schwarzschild-Str. 2, 85748 Garching bei München, Germany

e-mail: lmaud@eso.org

2 Leiden Observatory, Leiden University, PO Box 9513, 2300 RA Leiden, The Netherlands

3 INAF, Osservatorio Astrofisico di Arcetri, Largo E. Fermi 5, 50125 Firenze, Italy

4 Instituto de Astrofísica e Ciências do Espaço, Universidade do Porto, CAUP, Rua das Estrelas, 4150-762 Porto, Portugal

5 National Radio Astronomy Observatory, 1003 Lopezville Road, Socorro, NM 87801, USA

6 UK Astronomy Technology Centre, Royal Observatory Edinburgh, Blackford Hill, Edinburgh EH9 3HJ, UK

7 I. Physikalisches Institut der Universität zu Köln, Zülpicher Str. 77, 50937 Köln, Germany

8 Max Planck Institute for Astronomy, Königstuhl 17, 69117 Heidelberg, Germany

9 NASA Goddard Space Flight Center, Greenbelt, MD 20771, USA

10 Universidad Nacional Autónoma de México, Instituto de Radioastronomía y Astrofísica, Morelia, Michoacán 58089, Mexico

11 Department of Astrophysics/IMAPP, Radboud University, PO Box 9010, 6500 GL Nijmegen, The Netherlands

12 School of Physics and Astronomy, University of Leeds, Leeds LS2 9JT, UK

13 Anton Pannekoek Institute for Astronomy, University of Amsterdam, Science Park 904, 1098 XH Amsterdam, The Netherlands

14 Institute of Astronomy and Astrophysics, University of Tübingen, Auf der Morgenstelle 10, 72076 Tübingen, Germany

15 Max-Planck-Institut für Astrophysik, Karl-Schwarzschild-Str.1, 85748 Garching, Germany

16 Kapteyn Astronomical Institute, University of Groningen, The Netherlands

17 SRON, Landleven 12, 9747 AD Groningen, The Netherlands

18 European Southern Observatory, Alónso de Cordova 3107, Vitacura, Casilla, 19001 Santiago de Chile, Chile 


\section{Appendix A: Disc mass and other lines}

\section{A.1. Disc mass}

The disc mass is estimated in the standard fashion:

$$
M=\frac{g S_{v} d^{2}}{\kappa_{v} B_{v}\left(T_{d}\right)},
$$

where $S_{v}$ is the source flux, $g$ is the gas-to-dust ratio $=100$, $B_{v}\left(T_{d}\right)$ is the Planck function for a temperature, $T_{d}, d$ is the source distance, and $\kappa_{v}$ is the dust opacity coefficient. At $1.3 \mathrm{~mm}(220 \mathrm{GHz})$ we use $\kappa_{v}=1.0 \mathrm{~cm}^{2} \mathrm{~g}^{-1}$, as suggested for dust with thin ice mantles at densities of $10^{6}-10^{8} \mathrm{~cm}^{-3}$ (Ossenkopf \& Henning 1994). As noted, we use temperatures, $T_{d}$, between 50 and $150 \mathrm{~K}$. Even when temperatures lower than the disc average continuum brightness temperature are used, $193 \mathrm{~K}$ and $138 \mathrm{~K}$ dependent on the free-free subtraction, or temperatures that are found by scaling from the source temperature (where the inner radii are slightly hotter), the disc mass should be considered as a lower limit in the optically thick regime. An optical depth of $\tau=1$ would account for an increase in disc mass by a factor of 1.6 , to between 1.0 and $4.2 M_{\odot}$

\section{A.2. Other lines}

We detected the previously known strong SiO (5-4) molecular line emission and the $\mathrm{H} 30 \alpha$ radio-recombination line. The PV diagrams for these lines are shown in Fig. A.1. The $\mathrm{SiO}$ also traces the disc structure well, following the $\mathrm{H}_{2} \mathrm{O}$ emission at disc radii of $<120 \mathrm{au}$, but at low velocities closer to the source $V_{\mathrm{LSR}}$, the $\mathrm{SiO}$ is significantly more extended and reaches out to at least 250 au in radius before the surface brightness sensitivity is too low. The map was made with robust 1.5 , the resolution is $30 \times 24$ mas at a PA of $64.9^{\circ}$ and the sensitivity is $1.02 \mathrm{mJy}$ beam $^{-1}$ per $0.8 \mathrm{~km} \mathrm{~s}^{-1}$ channel. The extended emission was seen previously in our lower resolution ALMA observations (Maud et al. 2018) and is thought to be tracing outflowing material in a disc wind.

The $\mathrm{H} 30 \alpha$ emission appears unresolved for each channel in the image cube and only traces the very central region of G17.64, although there is a marginal shift in spatial position from blue- to red-shifted velocities. We imaged using a robust 0.0 to provide the highest resolution $\left(20 \times 18\right.$ mas, $\left.\mathrm{PA}=-88.4^{\circ}\right)$. The resulting sensitivity is $1.11 \mathrm{mJy}^{-1}$ beam ${ }^{-1}$ per $1.3 \mathrm{~km} \mathrm{~s}^{-1}$ channel. In the PV diagram, Fig. A.1b, the slight shift in position with change in velocity is clearer, and is in the same rotation sense as the $\mathrm{H}_{2} \mathrm{O}$ and $\mathrm{SiO}$ emission. The $\mathrm{H} 30 \alpha$ could be tracing a hot inner rotating structure inward of the molecular line region. We note that the total spectral coverage of our data are SPW0 216.746-218.621 GHz, SPW1 218.854-220.746 GHz, SPW2 230.938-232.812 GHz, and SPW3 233.048$234.94 \mathrm{GHz}$.
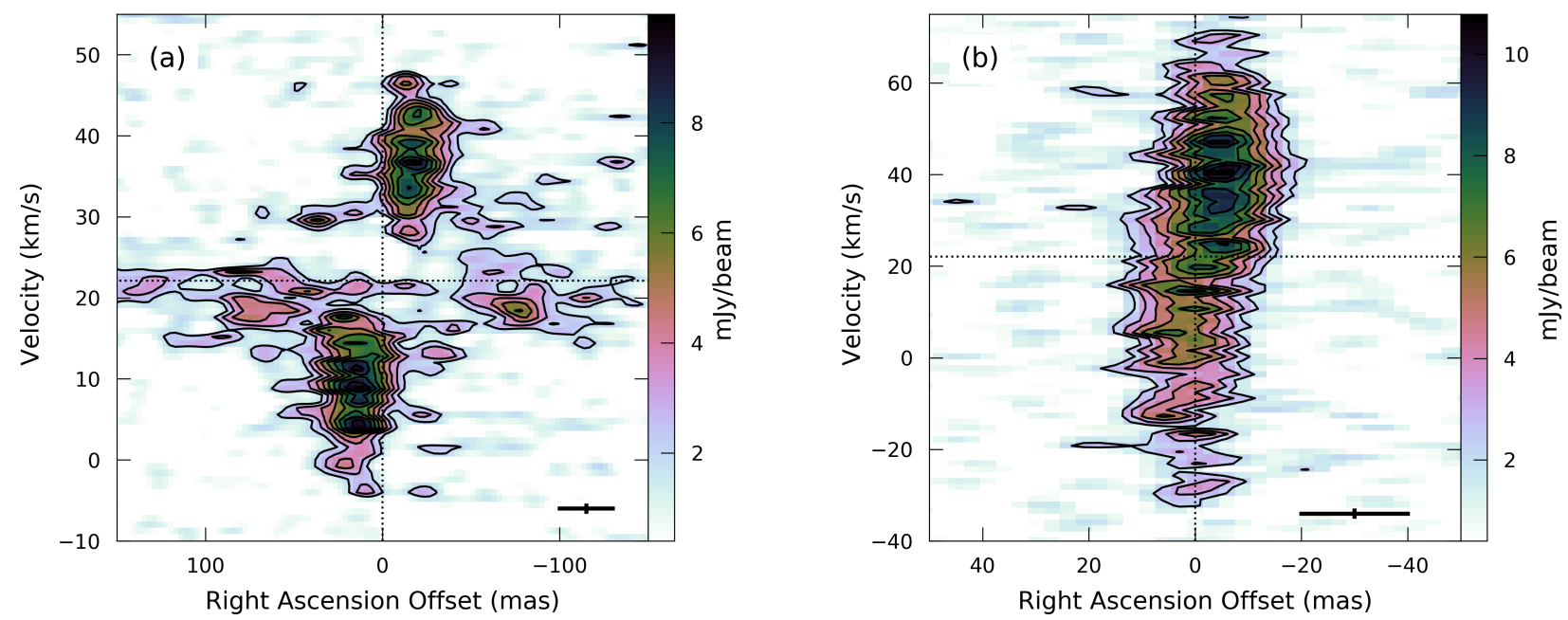

Fig. A.1. Panel $a$ : $\mathrm{PV}$ diagram for $\mathrm{SiO}(5-4)$ emission taken using the same cut $\mathrm{PA}$ and width as for the $\mathrm{H}_{2} \mathrm{O}$ presented in Fig.2b, a 5 pixel-wide slice along the dashed line at $\mathrm{PA}=25.9^{\circ}$. The resolution is shown at the bottom right as the beam major axis (30 mas) and the velocity resolution $\left(0.8 \mathrm{~km} \mathrm{~s}^{-1}\right)$. The inner structure from -50 to +50 mas overlaps that of the $\mathrm{H}_{2} \mathrm{O}$ emission, although we see emission extend past \pm 50 mas $(110$ au) at low velocities (cf. Maud et al. 2018). Panel b: same as (a), but showing the PV diagram for the H30 $\alpha$ radio-recombination line. The resolution is shown at the bottom right, representing the 20 mas beam major axis and a $1.3 \mathrm{~km} \mathrm{~s}^{-1}$ velocity resolution. Although tentative, there appears to be a very slight shift in position of the $\mathrm{H} 30 \alpha$ emission in the same rotation sense as the disc. It is possible that this traces very hot rotating inner material inward of the molecule line emission in the disc. In both panels the black contours of the data are plotted at the $20-80 \%$ levels in steps of $10 \%$. The axis scales and velocity range are different. 